\title{
AB0273 PERSONAL FACTORS IMPORTANT TO PEOPLE WITH RHEUMATOID ARTHRITIS AND THEIR COVERAGE BY PATIENT-REPORTED OUTCOME MEASURES
}

M. Dür ${ }^{1}$, M. Coenen ${ }^{2}$, V. Fialka-Moser ${ }^{3}$, A. Kautzky-Willer ${ }^{3}$, I. Kjeken ${ }^{4}$, M. Mattsson ${ }^{5}$, C. Boström ${ }^{6}$, J. Smolen ${ }^{1}$, T. Stamm ${ }^{1} .{ }^{1}$ Internal Medicine III, Division of Rheumatology, Medical University of Vienna, Vienna, Austria; ${ }^{2}$ Ludwig-Maximilians-University, Munich, Germany; ${ }^{3}$ Medical University of Vienna, Vienna, Austria; ${ }^{4}$ Diakonhjemmet Hospital, Oslo, Norway; ${ }^{5}$ Luleå University of Technology, Luleå; ${ }^{6}$ Karolinska Institutet, Stockholm, Sweden

Background: There is an increasing call to use patient reported outcome measures (PROMs) in health outcome research, because the perspective of patients is an essential part concerning the end results of health care. The coverage of patients' perspective by PROMs relevant in rheumatoid arthritis (RA) has been examined regarding all domains of the International Classification of Functioning, Disability and Health (ICF) except personal factors (PFs). Since the ICF did not classify PF, some researchers attempted to provide a classification of the PFs.

Objectives: We aimed to identify themes meaningful to people with RA, to determine which of these were attributed to PFs previously and to explore their coverage by PROMs.

Methods: We explored life stories to identify themes meaningful to people with RA and determined whether they have been previously attributed to PFs in the existing literature. Additionally we conducted a systematic literature search to identify PROMs relevant in RA. Finally, we explored whether the identified PROMs cover those themes which were attributed PFs previously.

Results: Twenty-two themes were found to be meaningful to 15 people with RA, of which 13 were attributed to PFs previously. Five themes were linked to activity and participation or environmental factors and four were not covered by the ICF. The systematic literature search resulted in the identification of 33 PROMs. Of these, the London Coping with Rheumatoid Arthritis Questionnaire and the Rheumatoid Arthritis Self-Efficacy Questionnaire covered most PFs. Examples of the coverage of themes attributed to PFs by PROMs are depicted at the Table 1.

Table 1. Examples of the coverage of themes attributed to PFs by PROMs

\begin{tabular}{lccccc}
\hline Themes & ASES & GSES & LCRAQ & RASE & WOC-R \\
\hline Coping & + & + & + & + & + \\
Involvement into disease management & + & & + & & + \\
Optimistic reflection & & + & + & + & + \\
Resilience & & + & + & + & + \\
Self-efficacy & + & + & + & + & + \\
\hline
\end{tabular}

Conclusions: The findings provide information about which PFs are meaningful to people with RA and could thus be targeted in non-pharmaceutical interventions. To our knowledge this was the first study exploring the coverage of PFs by PROMs relevant in RA. The exploration of the PROMs can support clinicians and other health professionals with the choice of which PROMs to use when developing or evaluating the effect of their interventions.

Disclosure of Interest: None declared

DOI: 10.1136/annrheumdis-2014-eular.3154 